\title{
Report Adverse Events
}

National Cancer Institute

\section{Source}

National Cancer Institute. Report Adverse Events. NCI Thesaurus. Code C128520.

The healthcare provider or setting must report adverse events of interest to the Manufacturer, MedWatch, and/or a REMS Program. 